ISSN: $1130-3743$

\title{
LOS PROCESOS FORMATIVOS Y EL SISTEMA EMOCIONAL ${ }^{1}$
}

\section{The emotional system and the educational processes}

\section{Le système émotionel et les procesus formatifs}

Joaquín García CARRASCO

Universidad de Salamanca. Facultad de Educación. Departamento de Teoría e

Historia de la Educación. Paseo de Canalejas, 169. 37008 Salamanca.

Correo-e: carrasco@usal.es

Fecha de recepción: enero de 2006

Fecha de aceptación definitiva: abril de 2006

BIBLID [(1130-3743) 18, 2006, 27-53]

RESUMEN

Se resalta en el documento la importancia del sistema emocional para el funcionamiento de la mente en su conjunto. Se muestra cómo esa importancia se hace evidente en las prácticas de las diferentes culturas y ocupa un lugar prominente en las narrativas donde se plasma, tanto la experiencia como el sistema simbólico. Aporta una serie de argumentos, convergentes en la dirección de que es posible la formación de la sensibilidad y, de hecho, ha sido una de las prácticas habituales en la historia cultural de la humanidad. Toma como metodología para el estudio del sistema emocional, tanto la hermenéutica de la comunicación como la investigación de los mecanismos corporales subyacentes. cional.

Palabras clave: emoción, sentimiento, pedagogía emocional, inteligencia emo-

1. Este trabajo se ha beneficiado de los comentarios y sugerencias de José Manuel Muñoz, miembro como yo del grupo de investigación "Procesos, espacios y prácticas educativas". También de los jueces que juzgaron el texto en vistas a su publicación y de otros compañeros del área de conocimiento "Teoría e Historia de la Educación", que tuvieron a bien leer el documento, aconsejando correcciones, precisiones y supresiones.

(c) Ediciones Universidad de Salamanca

Teor. educ. 18, 2006, pp. 27-53 


\section{SUMMARY}

The document highlights the importance of the emotional system for the operation of the mind in its entirety. It shows how this significance becomes evident in the practices of different cultures and occupies a prominent place in the narratives where both the experience and the symbolic system are given expression and in fact, it has been one of the habitual practices in the cultural history of the humanity. It argues that it is possible to teach sensibility. The methodology includes for the study of the emotional system both hermeneutics of communication and the study of the underlying physiologic mechanisms.

Key words: emotion, sentiment, emotional pedagogy, emotional intelligence.

\section{SOMMAIRE}

Le document met en relief l'importance du système émotionel pour le fonctionnement mental global. Il montre comment cette importance se fait évidente dans les pratiques des diférentes cultures. Le système émotionel occupe une place importante dans les écrits, dans le vécu personel et dans le symbolique. Il aporte une série d'arguments convergeants démontrant que la formation de la sensibilité est possible et, en fait, elle à été une pratique habituelle dans l'histoire culturel de l'humanité. Il prend comme méthodologie de l'étude du système émotionel, tant l'herméneutique de la comunication, que l'investigation des mécanismes corporels sous-jacents.

Mots clef: émotion, sentiments, pédagogie émotionelle, intelligence émotionelle.

\section{INTRODUCCIÓN}

Las páginas de este documento únicamente pretenden aportar un marco general en el que situar los trabajos sobre aspectos particulares que le siguen en este número monográfico y un contexto en el que se muestre la convicción cultural de que es posible planificar la intervención formativa, teniendo en cuenta el estrato emocional y que, de hecho, esa intervención ha constituido una parte importante de las prácticas en todas las comunidades culturales a lo largo de la historia. Por lo tanto, podríamos decir que una parte importante de la pedagogía implícita acumulada en la cultura responde a la gestión comunicativa de prácticas orientadas a la conformación del sistema emocional. Cosa que no siempre se ha puesto de relieve, subrayando la importancia que le corresponde. Incluso, a veces, resaltar este cometido ha sido motivo de cierto menosprecio, como un dedicarse a las "partes blandas" de los quehaceres de la formación. 
Como autor que ha escrito sobre la "pasión" en la enseñanza y el aprendizaje, me he sentido a menudo como un exiliado, un vagabundo por la periferia de la Academia... (R. Fried, 1995) ${ }^{2}$.

Al decantar la enorme producción bibliográfica actual sobre las emociones, tal vez el principal sedimento obtenido se concrete en el papel fundamental del sistema emocional en el funcionamiento del psiquismo humano, frente a quienes lo piensan o pensaban como mero complemento o tonalidad de la actividad cognitiva.

En las sociedades complejas, la inteligencia queda preferentemente asociada a la competencia en la resolución de todo tipo de problemas técnicos. J. Bruner califica esta concepción descriptiva de las acciones inteligentes como modelo de la inteligencia paradigmática. Advierte, enseguida, que si uno atiende a las conversaciones y a los escenarios en los que se expresa el que califica de modelo de la mente narrativa (Bruner, 1988), aquella en la que los seres humanos practican la competencia interactiva y la comunicación en vistas al mutuo entendimiento, los sentimientos son actores siempre presentes en las temáticas y timoneles que gobiernan las orientaciones de la persona, tanto en el momento de la toma de decisiones (Damasio, 1996), como a medida que el sistema persona y la identidad personal se van construyendo (Damasio, 2001) y como componente fundamental en toda experiencia vital (Damasio, 2005). Siendo tan importantes los fenómenos emotivos, y tan habituales en los trasiegos comunicacionales de experiencia, sin embargo, tal vez sean los fenómenos peor conocidos por la ciencia de cuantos da muestra la mente. Especialmente, son aquellos para los que la geografía lógica de los conceptos empleados se encuentra peor señalizada (Ryle, 1967).

Las aportaciones de los científicos coinciden con la experiencia de los educadores, incluso con la experiencia reflexiva de cada persona particular: el estado emocional interviene en los procesos de atención y percepción, facilita, dificulta y marca los procesos de memorización, interfiere en los de razonamientos y toma de decisiones, aporta significado y sentido a las relaciones interpersonales, y da forma a lo que el sujeto recoge como estado de su propia identidad en cada momento y en cada escenario de la vida cotidiana (Duch y Mèlich, 2005). Frente a quienes subrayan la condición de distorsionadores de la actividad de la mente, las emociones y los sentimientos funcionan, ante todo, como auténticos factores adaptativos que ocupan un lugar esencial y central en la regulación de las formas más elaboradas de la experiencia humana" (Speranza, 2003, 66). También hay procesos cognitivos distorsionantes de la percepción y la comunicación, como el prejuicio, sin que por ello minusvaloremos el poder de la cognición para la configuración de la acción y la comunicación. 
LOS SERES HUMANOS SON ANIMALES SENTIMENTALES

Para comprobar la trascendencia de este carácter aportaré unos cuantos argumentos.

\section{La tecnologia busca emular las emociones}

En el año 2001 se celebraron unas Jornadas sobre "Emoción y Conocimiento", organizadas por el Museo de la Ciencia de Barcelona (Morgado, 2002). En ellas R. Picard (2002), que ya había escrito un libro sobre Ordenadores emocionales Picard, 1998), reflexionaba de nuevo sobre el tema. Confesaba que en el MIT se empezaba a tomar en consideración que "la inteligencia involucra habilidades sociales y emocionales"; los ingenieros habían intentado dotar a los ordenadores de habilidades perceptivas; Rosalind Picard pretendía dotarlos de habilidades emocionales, incluso de habilidades para el reconocimiento de las emociones de los usuarios, especialmente la frustración. Fundaban sus investigaciones en la evidencia de que algunas emociones fundamentales - también denominadas primarias, por su condición de preprogramación innata-, presentan patrones expresivos modulados en el sistema motor, especialmente por los músculos de la cara, discriminables por un observador y con patrones faciales universales (Ekman, 2004). Las tres emociones que el ordenador programado consiguió reconocer con más seguridad fueron alegría, tristeza e ira. Pero, todavía no saben los ingenieros que parámetro medir, si lo hay, para discriminar en cada momento la emoción que el cuerpo expresa. El cuerpo, para muchos de sus cambios fisiológicos, proporciona señales observables e identificables desde el exterior. Acontecen algunos de esos cambios en paralelo con estados emocionales, como si de un marcador somático emocional se tratara (Damasio, 1996). Pudieron investigar los ingenieros características expresivas emocionales en el transcurso de la actividad, p. e., de un director de orquesta (Picard, 2002, 180), o refinar con la ayuda de la computadora la observación del comportamiento emocional de niños autistas, para mejorarlo. Dado que los usuarios se enfadan con el ordenador, éste, al reconocer el patrón, podría disculparse, si pudiera notar indicios inequívocos del enojo. No sólo reconocer, también los ordenadores podrían simular estados emocionales, los ingenieros podrían conseguir que se comporten como si tuviesen emociones: presentando una semiótica emocional, como el icono de sonrisa de los Mackintosh; disponiendo de reacciones rápidas de tipo primario, p. e., al detectar una situación de funcionamiento incorrecto, como en el caso de los ordenadores "Tándem"; mostrar una "reacción cognitiva" (informática), evidentemente sin respuesta fisiológica, por aplicar reglas lógicas a situaciones de "comprensión emocional" (¿?) creadas en la máquina, p. e., cuando un ordenador, que está realizando una operación importante, muestra un "emoticón" (signo gráfico) si el usuario pretende interrumpirla (el ordenador suple la emoción, mediante una señal que muestra una "inferencia lógica" respecto a la acción del usuario). 
Estas categorías de procesos informáticos pueden implementarse mediante procesos que simulan una secuencia emocional ${ }^{3}$. No se pueden plantear equivalencias informáticas con lo que denominamos experiencia emocional -porque implica conciencia-, ni con la psicofísica de la emoción - porque los componentes de la computadora no son biológicos-, ni con la comprensión de las emociones - porque supondría atribuir a la máquina conciencia intencional y teoría de la mente- (Trappl, 2003). En todo caso, la tecnología intenta tomar el sistema emocional como metáfora para mejorar la construcción de las computadoras. En el fondo, se busca emular el contenido de esa metáfora, porque se advierte que la inteligencia humana afronta problemas que los ordenadores no pueden acometer, especialmente aquellos que se plantean y se resuelven dialogando, los que se originan en las prácticas sociales de la competencia interactiva y que vitalmente requieren de la impregnación emocional. Son los seres humanos los que, siendo sistemas con conciencia intencional, constantemente procesando información, además de todo eso, son profundamente sentimentales. El sentimiento conlleva componentes cognitivos, componentes evaluativos, componentes vinculativos (afectivos), componentes expresivos (cambios en la apariencia) y componentes comprensivos (de los estados emocionales propios y de los demás), independientemente de la cultura considerada; se trata de funciones que corresponden a la condición humana, aunque podamos rastrear su historia evolutiva.

\section{Los seres bumanos sienten, expresan y comprenden las emociones}

La historia evolutiva evidenció la ventaja, desde hace muchos millones de años, de que los organismos dispongan de una estructura para desatar episodios emotivos, para sentir la conmoción interior, cuando sus sistemas "notan" o "actúan" y para, siquiera en el género Homo, vivenciar en la conciencia las experiencias emocionales y decidir mejor. Entre los caracteres biológicos recibidos por evolución, que pueden rastrearse en sus manifestaciones desde hace por lo menos 600 ma, y pueden comprobarse sobradamente en muchas especies, se encuentra una gama de emociones, esenciales para la subsistencia (Matignon, 2005): la sorpresa, el miedo, la agresividad, la tristeza, el asco; emociones que por este origen genético-evolutivo suelen calificarse de "emociones primarias". El sistema emocional, productor de correlatos y señales corporales, es nuestro vínculo evolutivo con otras muchas especies, el lazo de hermandad más aparente con otras culturas distantes y el puente sólido con el que se supera la distancia evolutiva entre el niño y su madre. Antes de que el bebé adquiera el habla, participan ambos del "lenguaje" compartido de las emociones y los sentimientos; vigila el niño, guiado por patrones innatos, las expresiones de los próximos, buscando establecer, mediante la "sincronía de

3. www.media.mit.edu/affect. 
estados emocionales", una interacción sensible que alimente el sistema persona y prepare los cimientos para una comunicación simbólica personalmente estructurada (Lewis, 2001, 76). En los seres humanos, sentir y experimentar emociones constituye un componente esencial de nuestro modo de proceder en el trato con el mundo, el procedimiento primario para predecir y elegir la respuesta comportamental más adecuada, el sistema primordial de evaluación de situaciones y de vinculación a "objetos de deseo", en términos freudianos. Los seres humanos practican elecciones, plantean previsiones y sacan consecuencias, a partir de las experiencias emocionales propias y de las expresiones emocionales de otros. Tal vez, para el intercambio emocional la humanidad de todas las culturas hable un lenguaje común, cuyas unidades básicas de significado fueron configuradas con anterioridad a la aparición misma del lenguaje. Y, tal vez, el hecho de que el lenguaje tome cartas en el asunto, al producirse la deriva divergente y las variedades de las lenguas, introduce distancias sentimentales aparentes donde, en el fondo del "corazón", estamos mucho más hermanados.

Que un lenguaje o idioma concreto tenga una palabra para expresar una emoción, es algo que depende de la habilidad del traductor y de las peculiaridades de la gramática, así como de la historia. Un lenguaje acumula un amplio vocabulario, en el cual figuran palabras, para expresar emociones, siempre que haya tenido artífices de la palabra influyentes, haya estado en contacto con otros idiomas, disponga de reglas para formar nuevas palabras... Cuando un lenguaje ha carecido de estos estimulantes, las personas describen cómo se sienten con circunloquios, metáforas, metonimias y sinécdoques (Pinker, 2000, 472).

Esto justifica que, aunque la comprensión sea suficiente (eficaz) para el mutuo entendimiento en la situación, el lenguaje se presente gelatinoso y con cierta ambigüedad, a la hora de discriminar y afinar el significado de los estados emocionales, a través del análisis del vocabulario. Ante todo, podemos afirmar que las emociones, en aquellas especies que las muestran, tienen la condición de procesos vitales, de patrones de acción vitalmente necesarios para la supervivencia. Entre los primates y entre los seres humanos, el contacto íntimo y empático, p. e., con la madre y con los hermanos, la sintonía emocional con el grupo, con la pareja y con otros congéneres, es tan esencial para la maduración, como para la reproducción. Normalmente, construimos la historia de las culturas y de las comunidades humanas recurriendo a condicionamientos sociales, económicos y técnicos, porque podemos presentar evidencias que apoyen la argumentación. Éste es un modo oficial de narrar la historia. Pero, si uno se sale de la perspectiva y se sitúa en la derrota que marca el sistema emocional, entonces descubre la importancia histórica de los "lazos vitales", entiende que "una pieza fundamental entre estas fuerzas sociales la integran las formas que tienen las personas de cuidarse entre sí y atender a las necesidades mutuas. Una temprana relación afectuosa y nutriente, como la que suelen disfrutar las madres con sus hijos, es tan vital para el desarrollo como lo es el calcio para los huesos" (Taylor, 2002, 8). Incluso se extiende la opinión, 
apoyada en cada vez más sólidos argumentos, de que la pegajosidad emocional -la dependencia social y toda su operatoria de estrategias y tácticas, vinculada a las prácticas propias de la inteligencia social-, fue decisiva para el desarrollo evolutivo del cerebro que llevó a la aparición de la especie humana (Humphrey, 1995; Ornstein, 1994; García Carrasco, 2004).

No obstante todos los datos que la epistemología evolutiva va proporcionando, los antropólogos han privilegiado en la descripción de la humanidad la inteligencia operatoria que expresa el manejo de herramientas; al hacerlo, han subrayado, con sesgo evidente, la importancia de la vía cognitiva que mostraba la inteligencia técnica ${ }^{4}$. Sin embargo, desde el alba de los tiempos, los humanos han dejado trazas y, con la llegada de la escritura, relatos muy avispados de inteligencia social y de la importancia que concedían a los comportamientos emocionales. Esos relatos y narrativas proporcionaron evidencia acerca de que comprendían la relevancia del estrato emocional, como parte fundamental de la inteligencia social de la que daban prueba. Aunque haya sido la calificación de racionales la dominante para tasar la diferencia entre los seres humanos y el resto de las especies, el modo racional de ser mentales, cada vez nos encontramos mejor equipados para poder sostener, que es aún más refinado, si cabe, lo que nos distingue en el mundo de la vida por nuestra manera peculiar de ser sentimentales.

\section{La metafórica cardiopática}

La cultura occidental dominante y académicamente más privilegiada identifica insistentemente actividad mental con procesos cognitivos (intelectuales). No obstante, la historia de la cultura proporciona evidencias de una amplísima identificación entre humanización del comportamiento (formación) y la brega del sistema emocional humano, una brega cultural constante entre calidades vitales y "cordialidades". En la epopeya sumeria de Gilgamesh, que suponen los eruditos antecedente de algunos pasajes bíblicos (el diluvio), una de las narraciones más antiguas conocida, el corazón está omnipresente en el documento como sede imaginaria de las emociones (Kramer, 1978). Son estos estados anímicos los que van marcando la historia de Gilgamesh, quien viajó para inspeccionar los confines del mundo y conocer sus secretos. Resalta el héroe en el poema por sus obras y por la grandiosidad de sus sentimientos, como el de amistad hacia Enkidu. G. Godwin (2004) proporciona un precioso elenco de documentos históricos donde el corazón es el protagonista, cuando la acción está bien planificada o cuando el sujeto se concentra

4. Recomiendo al lector, para completar esta indicación, la consulta del libro donde este tema se expande, dentro del mismo programa académico que conmemora el XXV aniversario de los Seminarios de Teoría de la Educación. Asensio, J. M.; García Carrasco, J.; Núñez Cubero, L. y Larrosa, J. (2006) La vida emocional. Las emociones y la formación de la identidad bumana. Barcelona, Ariel. 
en navegar por el charco de las emociones. También la autora indica, entre otras referencias, las más de mil que la Biblia hace al término corazón.

La literatura occidental propone con insistencia que la calidad del comportamiento es fruto de la bondad del corazón. La víscera cardíaca ha ocupado un lugar central del campo metafórico, de nuestro imaginario cultural, testimoniando la constante inervación del comportamiento por parte del complejo emocional; una especie de convencimiento cultural, probablemente universal, de que la regulación del comportamiento y su calidad relacional, la calidad de vida, la realización del ideal de vida humana, dependen de la orientación, el calibrado, la generación y la reparación de los sentimientos (Godwin, 2004). Tal vez sea éste uno de los campos más frecuentados por la literatura de todos los tiempos. El conjunto de la literatura podría entenderse, en este sentido, como una historia muy depurada, de constante reflexión, lingüísticamente mediada por el imaginario cardiopático; aparecería como un acercamiento a la comprensión del significado y relevancia de la sentimentalidad en la vida de los seres humanos, un convencimiento cienmilenario de la relevancia de la cultura de la sensibilidad. Más aún, las religiones, en sus partes no dogmáticas, constituyen auténticos tratados sobre la sensibilidad y la emocionalidad.

Muchos autores confirman la existencia de una amplia y extensa cultura de la compasión, de hecho denominan al budismo como la religión de la compasión infinita. Comentaba el Dalai Lama en un Seminario con científicos de variadas especialidades: "Las escrituras budistas hacen referencia a ochenta y cuatro mil tipos de pensamientos negativos y destructivos, que cuentan con sus ochenta y cuatro mil correspondientes antídotos" (Dalai Lama, 2004, 37). No deben espantar los números del catálogo, porque el estrato emocional posee una estructura que es capaz de multiplicarse indefinidamente. El objeto inductor sentimental puede ejercitar su provocación al menos por tres vías: (i) por la sensorial, (ii) desde la representación interior y (iii) por la evocación, recuperando la memoria de experiencias y representaciones pasadas. Se instituye, por estas vías, un sistema hipercompleto, hipercomplejo, de tramas emocionales. La experiencia de la emoción puede tornarse en experiencia emocional de nivel superior, los sentimientos conscientes, y generar metasentimientos, mecanismos reguladores éstos para el control y reequilibramiento de la vida emocional; los fallos en los mecanismos de control de estos niveles superiores pueden perpetuar la conmoción episódica de la emoción y crear el bucle vicioso del sujeto emocionalmente perturbado (Castilla del Pino, 2000, 33). La tradición budista ofrece una cultura emocional de más largo recorrido y mayor experiencia reflexiva que la cultura occidental, avezada en la discriminación escrupulosa entre el calibrado fino de la razón y el, aparentemente, más grosero y apresurado del estrato racional.

Las cumbres más altas del misticismo cristiano, San Juan de la Cruz (1993) como ejemplo, alcanzan sus niveles más excelsos desarrollando una interpretación teológica del Cantar de los Cantares de la Biblia, un libro sobre el amor. En todos los casos, tras la metafórica del corazón, aparece la práctica y la ascética del buen 
corazón, como un ejercicio sistemático y progresivo de discernimiento de valores en la vida humana. Se da a entender en esta tradición cardiopática que tanto el discernimiento como la discriminación no son funciones mentales meramente racionales, fundadas en razonamientos lógicos, sino funciones que se benefician también de los elementos vinculativos, valorativos y experienciales que se derivan de la actividad del sistema emocional.

Empiezan a aparecer, en la actualidad, historias que rastrean en el tiempo la dinámica cultural de los sentimientos: la historia cultural del llanto (Lutz, 2001), la fenomenología histórica del asco (Millar, 1998), la evolución del deseo como historia cultural de las estrategias para el emparejamiento humano (Buss, 1994) y ejercicios de hermenéutica de los sentimientos en circunstancias históricas de la humanidad (Zeldin, 1996), proponiendo otras maneras de interpretar y narrar la historia.

E. O. Wilson (1980) globaliza el papel de la emoción en la acción humana y en el proceso de pensamiento que la planifica: "Sin el estímulo y guía de la emoción el pensamiento racional se enlentece y desintegra. La mente racional no flota por encima de lo irracional; no puede liberarse y ocuparse sólo de la razón pura. Hay teoremas puros en matemáticas pero no pensamientos puros que los descubran".

Dado el lugar dominante que ocupan los estados emocionales en la comunicación oral y en la literatura de todos los tiempos y de todas las culturas, podría entenderse que la literatura contiene una bermenéutica de la emocionalidad, de la que podría discutirse el poder de resolución. La literatura se torna por esta vía una extraordinaria fuente de documentación. No otra cosa trata de demostrar el programa de investigación de que da cuenta M. Nussbaum (2005) en su obra El conocimiento del amor. Ensayos sobre filosofía y literatura.

Estimo que la advertencia inicial, para abordar el estudio del comportamiento emocional de los seres humanos, es la de no poner todo el reclamo en el vocabulario, dando por supuesto que las denominaciones al uso -emoción, sentimiento, afecto, pasión, entre otras-, son realmente certeras en sus discriminaciones, dentro de la experiencia narrativa del sujeto o dentro de los hábitos coloquiales de la comunidad; ni siquiera, en todos los casos, son precisas ni totalmente concordantes en la literatura especializada. Es fácil observar cómo esos términos, frecuentemente, son empleados con significados diferentes por distintos autores. Algunos defienden, con convicción, que el sentimiento es un estado emocional ecuánime, sin variaciones bruscas, estable y de baja intensidad, que no interfiere en la actividad racional; a diferencia del sentimiento, la emoción para esas personas es convulsiva, sobrecogedora, absorbente de la atención, desestabilizadora de la actividad atencional y discursiva. También hay sentimientos, como los del depresivo, que desestabilizan la mente. Como todos podemos comprobar, a poco que nos acerquemos con un mínimo rigor al vocabulario emocional, advertimos que no es su intención explicar, trata únicamente de compartir experiencias emocionales con sentido. En este quehacer, a pesar de la imprecisión terminológica, la comprensión arriba habitualmente a buen puerto o, al menos, con eficacia suficiente. 


\section{Del sentimiento al comportamiento ético}

La conexión entre sentimientos y vida moral la hemos comprobado en los reflejos culturales de la metafórica del corazón. Tal vez no haya autor más sorprendente e innovador en este punto que Spinoza (1632-1677), ni obra más cautivadora sobre el tema que su Ética (1988), sorpresa que ratifica A. Damasio, en su reciente obra En busca de Spinoza (2005), donde armoniza las tesis del viejo maestro con sus conclusiones más actualizadas sobre la neurofenomenología del sistema emocional. De esta relación entre sentimiento y moral elaboró una sistemática, todavía actual en muchos aspectos A. Smith (1723-1790) en su obra Teoría de los sentimientos morales (1997).

Muchos otros filósofos han desarrollado esta conexión. Un ejemplo reciente en aplicar la interacción entre la conducta moral y los sentimientos ha sido Hans Jonas. Cree el autor, entre otros argumentos, que el hecho de haber comprometido la estabilidad de la naturaleza con el poder de la tecnología obliga a la ética a cambiar e introducir nuevos imperativos. Valoraba el hecho de que la ética tradicional quedaba definida en los límites de la acción personal y sus consecuencias próximas; los riesgos globales de la naturaleza obligan a tener que asumir los humanos, por el principio de responsabilidad, consecuencias lejanas, efectos únicamente previsibles, futuros inciertos, la sostenibilidad de la biosfera, la calidad de vida para futuras generaciones, como criterios de valor moral para el comportamiento. Estas exigencias éticas le llevan a plantear que "...una relación afectiva con la comunidad, comparable al amor, cuyo destino él desea conducir hacia lo mejor [...], fundamenta aquella identificación emocional con el todo, el sentimiento de solidaridad. [...] El factum del sentimiento hace al corazón receptivo al deber (que de por sí no pregunta por eso) y anima con su impulso la responsabilidad asumida" (Jonas, 1995, 180).

En el proceso de la formación moral la generación del sentimiento garantiza la receptividad a los deberes que surjan de la deliberación moral sobre las situaciones consideradas. La beurística del sentimiento aparece como fundamento y garantía de lo segundo, la deliberación ética (Jonas, 1997, 2000). Victoria Camps $(2001,104)$ se encuentra convencida de que la moralidad necesita esa parte emotiva, la de sentir obligación. También está persuadida de que «los sentimientos se adquieren: nadie nace solidario, tolerante, o generoso". "Nadie nace... con actitudes y sentimientos morales: la solidaridad, la confianza, el respeto son sentimientos que se adquieren socialmente" (2001, 118). La ética en Schopenhauer, Horkheimer, Adorno y Levinas, y en tantos otros autores no seguidores de la teoría kantiana sobre la moral, es impensable sin el componente de los sentimientos 5 . La ética, en estos y en otros muchos autores, se presenta, en buena medida, como

5. La referencia a estos autores ha sido ampliamente resaltada entre los pedagogos españoles, con idéntica intención por P. Ortega, profesor de Teoría de la Educación en la Universidad de Murcia. 
un estudio sistemático de los sentimientos morales y una guía para la conformación de la sensibilidad.

Que los sentimientos están patentes en la práctica de la competencia interactiva, que las culturas expresen la importancia del sistema emocional en las prácticas comunitarias, son hechos demostrados. Pero, ¿son los sentimientos una dotación de la naturaleza que únicamente se expresa, pero sobre la que no se puede intervenir, cuyo perfil personal acompaña a cada sujeto del nacimiento a la tumba?

\section{¿SE PUEDEN FORMAR LOS SENTIMIENTOS?}

La respuesta a esta pregunta debiera constituir un capítulo importante de la teoría y la práctica reflexiva de la educación. Esta pregunta está en las miradas de los padres que ven a sus hijos descarrilar y en las de los educadores, que ven flacos de motivación a los estudiantes; está la pregunta en los corrillos sociales que ven escandalizados sucesos terribles promovidos con frialdad insolente, está en las lágrimas impotentes de todos los maltratados o en los que padecen escarnio y son marginados sin causa. La pregunta resume en una línea el mapa de trabajo para la elaboración de una pedagogía de la sensibilidad y también contiene el acicate para todo un programa de investigación. La pregunta plantea de manera directa si es posible o no a la cultura intervenir, interferir, actuar, en los procesos de creación, modificación, reparación, de la sensibilidad en general o en los procesos por los que se generan sentimientos particulares.

El proceso de construcción de la respuesta requiere de comprensión de las manifestaciones emocionales, implica la posibilidad de planificar y adquirir babilidades tutelares y actitudes solícitas, la de desarrollar empatía sentimental que establezca la sintonía imprescindible para que la interacción no se transforme, por culpa de los estados de ánimo, en un galimatías: la de poder reconstruir una comunicación emocionalmente distorsionada. Pretende, en última instancia, alcanzar explicaciones. Comprensión y explicación son hermanas que mutuamente se alimentan, por el intermediario de múltiples habilidades metodológicas, en el plano reflexivo y en el plano experiencial. La respuesta positiva, finalmente, se contiene en el convencimiento de que la mejora cualitativa de la comunicación y, en definitiva, la felicidad de poder dar de sí como todo un ser humano, es posible.

Si realmente son posibles las acciones formativas en el estrato de la sentimentalidad, esa posibilidad implica necesariamente la existencia de mecanismos que expliquen la generación-destrucción de sentimientos, a partir de acciones socialmente mediadas; si los mecanismos biológicos, culturales o sociales fuesen accesibles a las prácticas intersubjetivas, sería posible concebir procedimientos, tácticas comunicacionales, estrategias situacionales, que puedan estar al alcance de otros seres humanos que, por este motivo, cumplirán roles de educador. Tal vez no baste con intervenciones de personas en escenarios comunicativos, porque muchas veces las actitudes emocionales son resistentes al cambio mediante argumentos; 
quizás, la accesibilidad formativa al estrato emocional pudiera requerir, además, de un cambio global en el nicho convivencial, en vez de solamente acciones puntuales; o, quizás, lo uno y lo otro simultáneamente, una concordancia de acciones y situaciones acordes a la dinámica propia de la sensibilidad.

Éstos son componentes fundamentales del problema que nos hemos planteado y sobre el que están trabajando muchos pedagogos y otros profesionales en sus correspondientes campos de conocimiento. Para Spinoza en su Ética, el principio fundamental que debe dirigir en la práctica una pedagogía de la sensibilidad aplica el criterio popular "la mancha de la mora roja, con otra mora verde se quita". El principio espinoziano es que la neutralización de un afecto solamente se puede conseguir con otro afecto contrario y más fuerte. ¿Es esto posible, es ésta la única vía? Resumiremos una serie de argumentos que permiten confirmar que la educación de la sensibilidad es un proyecto posible y que la historia de la humanidad demuestra que ha sido una de sus prácticas habituales. Al describir los argumentos seleccionados, por considerarlos argumentos de mayor calado, se vislumbran sus implícitos programas o vías de acceso a la formación de la sensibilidad.

\section{Desalientos mal argumentados}

No podremos responder a la pregunta sobre si pueden ser formados los sentimientos, si no sabemos por lo que estamos preguntando ni tenemos idea de dónde queremos meter la mano. Ésta es una posición que se filtra muchas veces en textos y exposiciones. Lo que, en principio, es dificultad de comprensión del estrato sentimental humano, se transforma por vía de apresuramiento en fantasma y en sombras que mantienen desconcertados los proyectos. La experiencia de la humanidad muestra que sabe de lo que está hablando, es el método científico el que muchas veces se atora y no encuentra medio para construir, desde sus constricciones metodológicas, un modelo creíble.

Sin duda alguna, es casi imposible tratar de explicar qué es un sentimiento, cuál es su génesis, qué lo suscita, de qué factores personales y ambientales depende, cómo y por qué se extingue, etc. Nada de particular tiene que no resulte nada fácil conocer la afectividad propia y la de los demás y que, en consecuencia, se ejerza sobre ella tan escaso control (Polaino, 2003, 102) ${ }^{6}$.

En la lectura de muchos libros sobre el pensamiento y la cognición, dentro de la línea de investigación que se denomina programa cognitivista, puede uno concluir la lectura de un grueso volumen sin haber encontrado una sola vez la palabra emoción. Algunos autores lo confiesan con cierto arrepentimiento, resaltando

6. La cita corresponde al capítulo "La educación sentimental", en la p. 102. Trae el autor a colación un libro muy acorde con su punto de vista: MARINA, J. A. (1997) El laberinto sentimental. Barcelona, Anagrama. 
la importancia del asunto, aunque no se lo encontraron con su arado, en los territorios que andaban labrando.

¿Si no sabemos a lo que nos referimos en qué apoya la humanidad el convencimiento de que pueden y deben formarse? Lo que parece para todos aceptado es que los planes de formación en las instituciones de enseñanza se organizan, con criterio fundado y con evidencias de pedagogías reflexionadas, en lo que tienen de planificación cognitiva, pero solemos ser menos solventes y un poco más atolondrados en los planos en los que se ve directamente envuelta la sensibilidad: de ello deriva que el contexto actitudinal, el de los valores, los dominios estéticos e incluso la ética, frecuentemente estén faltos de plan o el más aparente no sea otro que el de empollar textos. No es verdad que la investigación no haya proporcionado materiales para calibrar mejor este asunto.

Una muestra etológica del cambio en la sensibilidad y de la mediación social que lo acompaña

Susan Mineka y sus colegas de Wisconsin comprobaron lo siguiente. Un mono rhesus hambriento criado en cautividad es capaz de pasar el brazo sobre un cajón, que contiene una serpiente, con el fin de obtener comida. Esto no tendría importancia si no se considerara que el miedo a las serpientes, a las aves rapaces y al felino constituyen instintos característicos, condicionamientos genéticos, de esta especie. Los adultos salvajes no alargarían nunca la mano, ni para coger comida estando hambrientos, si el brazo ha de pasar por la proximidad de una serpiente a la que han visto. Lo curioso es que si el monito ingenuo comprueba el acobardamiento de un colega en tal situación, también se asustará él la próxima vez. Este "aprendizaje del miedo", este aprendizaje de la condición de enemigo, culmina con éxito, incluso si la escena que contempla es una película. Pero, nunca aprendería por este medio el miedo a las pacíficas flores o a los inofensivos conejos (Slater, $2000,18)$. Estamos ante una emoción que encuentra su objeto por mediación social: el "aprendizaje" social de la emoción de miedo a las serpientes. Evidentemente, la predisposición al miedo a las serpientes es un rasgo de la especie, el disponer del aparato fisiológico de la emocionalidad es un producto de la evolución, guiado en la especie por su ADN. Pero el desencadenamiento de la experiencia de miedo respecto a ese enemigo ha sido, en este caso, consecuencia de un proceso de mediación social. Esto indica tres cosas: (i) el sistema está predispuesto a la activación; (ii) pero, dispuesto a hacerlo ante determinada categoría de representaciones y no ante otras; (iii) implica en la activación la mediación del comportamiento observado en otros de su misma especie o una experiencia personal dramática en relación con esos inductores. La disposición engatilla y engancha su objeto, por lo tanto, en un contexto socialmente mediado. Este caso demuestra que el aprendizaje de las emociones es, en alguna medida, posible y que no hay que esperar a la especie humana para comprobarlo. Podría fácilmente ampliarse la documentación etológica tanto 
en la dirección del componente emocional en los procesos de imitación, como en la de la mediación social en la construcción del valor emocional de las situaciones (Waal, 1993, 2002).

\section{Las emociones específicamente culturales}

El segundo argumento que aportamos tiene también mucho poder demostrativo. Nos referimos a la constancia de que existen emociones que son propias de comunidades culturales. Por lo tanto, su inducción forma parte de los procesos de formación socialmente mediada. Estamos ante la observación de casos de generación cultural de tramas o de configuraciones emocionales. Son muchos los estudios de antropología que dan cuenta de lo que denominaríamos emociones específicamente culturales.

Dylan Evans $(2002,31)$ describe la que nombra como "condición del jabalí" entre los gururumba de Nueva Guinea; describe el autor la complejidad de esa trama emocional, la representación que de la misma se forman los indígenas, los rituales dentro de los cuales se induce y la valoración cultural que de estos estados plantea la comunidad. En muchas culturas se encuentran términos de referencia emocional intraducibles que referencian tramas emocionales características: el amae japonés, la saudade en Portugal, la morriña en Galicia, la gana del español. En todos los casos se alude a un patrón emocional culturalmente condicionado.

D. Rougemont investiga lo que considera un rasgo de la cultura en Occidente, el conflicto entre la pasión amorosa y la institución matrimonial. Al hacerlo cree comprobar que el "Amor pasión" nace en el siglo XII. Con ello se quiere indicar la tesis de que los procesos emocionales transcurren culturalmente condicionados; o su equivalente, las tramas emocionales se individualizan en las culturas.

...yo no me propongo considerar el Tristán como una obra literaria, sino como un prototipo de las relaciones entre hombre y mujer dentro de un grupo histórico dado: la élite social, la sociedad cortesana y caballeresca de los siglos XII y XIII $(1993,20)$.

La Ilustración ha sido abundantemente estudiada. Muchos la presentaron como un siglo que cultivó a palo seco la razón. Una amiga de malogrado talento me advertía, por el contrario, que el "logo" del siglo representaba una diosa bien dotada y sensual. J. Seoane Pinilla, investigador concienzudo, señala un hilo de investigación que finalmente formula y comprueba la siguiente hipótesis:

En el siglo XVIII se inventan los sentimientos que hoy tenemos y que éstos han servido de componente esencial para construir nuestro presente ético y político (2004, XIII).

Generalizando el planteamiento, hay autores que defienden la postura de que, desde su unidad psicosomática, el "hombre vive afectivamente en el mundo" y 
que, por lo tanto, es ese dominio vital donde las emociones toman cuerpo y toman identidad. No existirían emociones crudas, sino cocidas culturalmente.

El alejamiento en el espacio, por los datos etnológicos, o en el tiempo, a través de la historia de las mentalidades, subraya las formas cambiantes y las convencionales que toman las emociones y sus puestas en escena, según los grupos sociales y las circunstancias (Breton, 2004, 131).

Lo tienen claro estos autores, más de uno cree poder afirmar que estas transformaciones culturales de las emociones se encuentran en el origen del género literario de la novela; porque, por esta vía literaria, encontraban el aliviadero expresivo para poder tratar culturalmente con ellas. La filosofía se quedaba, como marco narrativo, un tanto estrecho. M. Nussbaum (2005) proporciona argumentos para demostrar la dependencia entre la posibilidad de exploración de determinadas experiencias humanas, entre ellas las del amor, y su dependencia del género narrativo que emplea el explorador.

Ahora bien, si la hipótesis de que los sentimientos pueden ser formados, que puede darse planificación de la educación de la sensibilidad, se considerase confirmada, el corolario inmediato es el de que tales tramas emocionales, para continuarse en la comunidad, han de encontrar medio para su incorporación. Desde ese preciso momento, quedaría postulado el sentido de la educación sentimental. La propuesta encontró como nicho expresivo el propio género literario de la novela en G. Flaubert (1990) y lo ha encontrado, también, en el género del ensayo filosófico, como en el caso de J. Marías (1992) y el varias veces referido de M. Nussbaum. Todo ello induce a pensar que en la aproximación a estos modos de narrativa cabe la posibilidad de elaborar planes de alfabetización emocional, como medio pedagógico para la comprensión profunda del sentido de muchas de esas narrativas.

\section{La inteligencia emocional}

No sólo hablan a favor de la educabilidad de los sentimientos los argumentos anteriores. Hoy se postula tal posibilidad como una práctica habitual y propia de una aptitud específica de la mente humana. Tal vez uno de los libros más leídos y comentados sobre el sistema emocional sea el de D. Goleman sobre Inteligencia emocional (1996). Este libro arranca su recorrido a partir de la constatación de que, en este momento cultural del mundo, sobresale el rasgo del "malestar emocional", socioculturalmente inducido. Pero, al mismo tiempo advierte que el psiquismo humano dispone de una aptitud vital, justamente denominada inteligencia emocional, como sistema gestionador de los sentimientos, del carácter y de los impulsos afectivos e, incluso, morales. Podemos, mediante esta aptitud,

tomar las riendas de nuestros impulsos emocionales, comprender los sentimientos más profundos de nuestros semejantes, manejar amablemente nuestras relaciones o desarrollar lo que Aristóteles denominara la infrecuente capacidad de "enfadarse 
con la persona adecuada, en el grado exacto, en el momento oportuno, con el propósito justo y del modo correcto" (Goleman, 1996, 15).

La versión inglesa del libro (1995) y una portada del TIME con la pregunta "What's your EQ?, popularizó el tema y situó al libro a la cabeza de los más vendidos. Sin embargo, únicamente mostraban la punta de una línea de investigación. R. Thorndike, mucho antes, había planteado la hipótesis de una inteligencia social"; quedó reforzada la idea, en los años 90, con la propuesta de H. Gardner (1994) sobre "inteligencias múltiples" (Frames of Mind), en cuyo catálogo incluía una "inteligencia interpersonal" que, entre otras cosas, gestionaba emociones y afectos. Entre las funciones de esta estructura mental se encontraban la competencia en el acceso a la identidad de los propios sentimientos, la capacidad de discriminación, la aptitud para categorizarlos en códigos simbólicos y la de gestionarlos para organizar la conducta. Suponían estos autores que no quedaba completo el inventario de funciones mentales con lo planteado dentro del modelo de la inteligencia paradigmática.

Estaríamos ante un sustrato mental, para un componente esencial de lo que muchos sociólogos denominan entendimiento social, muchos psicólogos competencia interactiva, y los pedagogos proceso de socialización. Sin la aptitud flexible para el manejo eficiente del sistema emocional, probablemente la formación de la mente humana sería imposible. P. Salovey ha sido uno de los investigadores que más han afinado sobre el tema y probablemente el primero en introducir el concepto de inteligencia emocional en el campo de la psicología. Para él,

El concepto de inteligencia emocional aísla un conjunto específico de aptitudes que se hallan implícitas dentro de las capacidades abarcadas por la noción de inteligencia social (Grewal y Salovey, 2006).

Estas investigaciones han quedado reforzadas con las de A. Damasio (2005), entre otros, confirmando la interrelación esencial y la inseparabilidad estructural entre la actividad del sistema emocional y la que corresponde al razonamiento ${ }^{7}$. Salovey y Mayer propusieron un modelo para el análisis de la inteligencia emocional que incluye cuatro dominios: (i) capacidad para identificación de las emociones; (ii) capacidad para el aprovechamiento de la información emocional, de manera que faciliten los procesos de deliberación y razonamiento; (iii) capacidad de comprensión de las narrativas emocionales (expresión emocional), fuente para

7. Uno de los jueces de cuyo criterio se ha beneficiado este artículo hace referencia a unas declaraciones de A. Damasio en el periódico La Vanguardia (29-III-2006). En ellas se reiteraba Damasio en que «hemos puesto el énfasis en el proceso cognitivo y hemos marginado las emociones, ique son decisivas! Por eso hoy estudio la neurobiología de emociones y sentimientos". Para señalar más adelante: "Una emoción es un cambio que se produce en tu cuerpo y en tu cerebro. Y un sentimiento es la idea que tienes de ese cambio. ¡Una idea, pues, es una representación! La representación de una emoción”. De aquí no se deduce que Damasio piense que el sentimiento es como cualquier otro pensamiento. El 
la "estratégica emocional"; (iv) capacidad de control emocional, en sí mismo y en los demás. Fundados en este modelo, Mayer-Salovey-Caruso, diseñaron el MSCIT, test que, mediante informes sobre tareas, facilita el estudio de las competencias emocionales de los sujetos (Grewal y Salovey, 2006). Un instrumento popularizado para construir criterio acerca del nivel de desarrollo alcanzado en esos niveles de aptitud (Cociente emocional), es el propuesto por S. Matineau (1997).

El fracaso en la formación de la inteligencia emocional, la mala educación emocional, no se mide en términos de error o de ignorancia, sino en magnitudes de autoengaño (Goleman, 1997). Cree Goleman que ya estamos en condiciones "académicas" - conocimiento disponible sobre los mecanismos del sistema emocional-, para introducir en el diseño de planes formativos, prácticas para el fomento de la empatía y, en general, de la inteligencia emocional (Goleman, 1999), o para la creación de dominios, ecológicamente correctos, para la dinámica positiva de las experiencias emocionales (Soler, 2003).

Son muchas las críticas al constructo inteligencia emocional (Aguado, 2005), incluso se discute si sus referentes no están ya contenidos en otros que la psicología y la pedagogía manejan. Sin embargo, cierta convergencia de opinión es patente, acerca de que una inteligencia concebida meramente como procesadora de información es un modelo incompleto e insuficiente para integrar el conocimiento que vamos adquiriendo acerca de los mecanismos del sistema emocional. Ésta es la cuestión precisa que asume como investigación interdisciplinar el "Mind and Life Institute" de la Universidad de Harvard. Las investigaciones de sus participantes no dejan dudas acerca de que una de las tareas que la especie humana viene asumiendo, dentro de su aptitud para la incorporación cultural al entendimiento social, y como rasgo de la condición humana en el mundo, para poder vivir, ha consistido en la cultura emocional (Goleman, 2003).

Creo que la práctica realizativa de esta competencia interactiva en el manejo del sistema emocional ha constituido, dentro de las narrativas cardinales de todas las culturas, en todas las épocas, un eje esencial de lo que se dio en llamar "Sabiduría". No le es imprescindible un grado excelente de inteligencia paradigmática ni una acendrada cultura académica. Reconocía J. Saramago, en su discurso de aceptación del Premio Nobel, que su abuelo analfabeto fue el hombre más sabio que él había conocido.

\footnotetext{
sentimiento de tristeza consistiría, en ese caso, en pensar en el motivo por el que se tuvo la emoción correspondiente. El sentimiento incluye la conciencia y la representación del estado del sujeto dentro de una experiencia subjetiva en la que interviene para su configuración lo que Damasio denomina "el marcador somáticon. El sentimiento aparece en sus obras como un estado complejo, de nivel superior (implicación cortical), conformado por la conciencia, la representación y el estado emotivo. Sin cualquiera de esos componentes la estructura compleja del sentimiento se destruye.
} 


\section{La calidad en la deliberación para el fomento de la inteligencia emocional}

La práctica filosófica, en tanto que ejercicio reflexivo sobre las formas de representación y comprensión del mundo y de la vida de los seres humanos, fue muy temprano requerida para dar cuenta y proponer orden en las turbulencias emocionales. Éste es uno de los ejes desde los que interpretar la filosofía helenística, llevada tempranamente a prestar atención a los males de la sociedad.

Uno de los logros más impresionantes de la filosofía es haber mostrado irrefutablemente y con detalle cómo las condiciones sociales conforman la emoción, el deseo y el pensamiento (Nussbaum, 2003, 31).

Hasta el punto de que M. C. Nussbaum afirma que son las propuestas de "terapias filosóficas" las que llevaron a los filósofos helenistas a tomar como objetivo la contrucción de enfoques conceptuales acerca de las prácticas educativas. Algo que Epicuro formulaba, dentro de lo que podría denominarse modelo médico, como la terapia social y personal mediante el argumento. Algunos antiguos sistemas filosóficos tomaron las pasiones como uno de los ejes de su indagación, especialmente los estoicos. Crisipo, discípulo aventajado, compone un tratado cuyo título lo dice todo, Therapeutikón o Etbikón, una propuesta práctica con que curar las enfermedades del alma, sus discapacidades (Zamora, 2003). Independientemente de lo que entendieran por pasión y por emoción, y de cómo se valoren unas y otras, lo que dejan claro los estoicos es el convencimiento racional de que hay manera de doblegarlas, de conducirlas, de apagarlas, de reorientarlas o de alimentar su voraz garganta incendiaria. La filosofía nunca abandonó la ética, ni ésta dejó de lado jamás tomar partido en la puesta en orden de los sentimientos, con lucidez dispar; en esto, Spinoza, refutando a los antiguos estoicos, sigue siendo una lumbrera.

También hoy, muchos filósofos se han visto interpelados por el malestar emocional de la cultura. Desde el 2002 existe la "Asociación Española para la Práctica y el Asesoramiento Filosóficos" (Asepraf), miembro de la IGPP (Internationale Gesellschaft für Philosophische Praxis), fundada en 1981; su actividad de asesoría se funda en la suposición de que detrás de muchas dificultades vitales, como la "emotividad desbordada", existe el mecanismo de "hábitos irracionales de pensamiento y un diálogo interno plagado de juicios valorativos" incorrectos (Caballé, 2004, pássim); argumentan sus socios que, si ésta es la causa (el mecanismo, el modus operandi), el asesoramiento filosófico que corrija los sesgos del pensamiento mejorará la dinámica emocional (Marinoff, 2000). Proponen la sugerencia general de que la deliberación sobre un texto filosófico, bueno y apropiado, pueda tener efectos benéficos sobre la dinámica sentimental.

La deliberación sobre los sentimientos, cada vez es propuesta desde más fronteras de conocimiento, por considerarse que los trastornos emocionales se han instituido en un rasgo muy visible de la etiopatogenia del mundo desarrollado. 
Los estudios clínicos sugieren que entre el 50 y el 75 por ciento de todas las visitas al médico están motivadas sobre todo por el estrés, y que, en términos de mortalidad, el estrés es un factor de riesgo más grave que el tabaco (Servan-Schreiber, 2004).

Ya se interprete el estrés como (i) amalgama de cambios generales e inespecíficos del organismo, como respuesta a la influencia de determinadas situaciones, el precio que paga el cuerpo por la tensión de la vida; (ii) ya se mire como sindrome general de adaptación o (iii) como agotamiento de la capacidad de respuesta a las demandas conductuales de la situación (Gil-Roales, 2004); en todos los casos, cursa el proceso de estrés con un ánimo esquilmado; es decir, están implicados estados emocionales. Precisamente la mediación emocional es considerada por muchos como la vía por la que el estado de estrés adquiere responsabilidad en la afectación de la salud. De ahí que la sanación de estos estados de agotamiento, o los de depresión y ansiedad, por no indicar otros, implique la posibilidad, directa o indirecta, de cambio en los estados emocionales. Se ha reconocido la capacidad curativa de la palabra en el psicoanálisis (algunos consideran el psicoanálisis como una teoría general de las emociones), la de medicamentos tan conocidos como Prozac y toda una amplia panoplia de prácticas de medicina alternativa.

Es difícil demoler este tren de argumentos, cuya lista podría agrandarse e incluso cada argumento expandirse, acerca de la posibilidad de la formación, de la planificación de la acción de formación, con el objetivo de allegar calidad de vida emocional a los seres humanos. Como también es difícil de doblegar que en el interior de ellos aparecen convencimientos acerca de "estrategias" formativas para el dominio de la sensibilidad. Como demuestra M. C. Nussbam $(2003,113)$ respecto a Aristóteles, el sistema emocional forma parte de la conciencia intencional, de la manera como el ser humano establece sus relaciones vinculativas con el mundo de las cosas y de los seres humanos. También desde la filosofía helenista se plantean los problemas entre cognición y emoción, entre sentimiento y creencia, con interpretaciones dispares y sin que definitivamente quede claro, si entre las vías de acceso a la formación de la sensibilidad hay otras que no sean el de proponer tan sólo un cambio en las creencias, un cambio en las convicciones por la vía argumental. La experiencia acumulada demuestra que hay más, que tiene que haber más porque, al menos en el campo de las actitudes, siempre se ha recalcado que el componente cognitivo que las constituye, por estar muy "cristalizado", es resistente al argumento. De ahí la importancia a conceder en este campo a "artes" como el teatro, la práctica, la música, el juego, la cinematografía... Comentaba Leontiev en el prólogo a la obra de Vigotsky Psicología del arte (1972), que para este autor el arte constituía "una técnica de las emociones". 


\section{ENCONTRAR EL CÓMO ACTUAR Y LA DIFERENCIA ENTRE COMPRENSIÓN Y EXPLICACIÓN}

\section{La comprensión y la bermenéutica de los acontecimientos}

El discurso pedagógico se compone de proposiciones, relativas a diferentes categorías de procesos y sus interdependencias; se podría pensar que es mejor expresión la de que el discurso pedagógico tiene que ver con acontecimientos, y con interferencias en acontecimientos; es decir, con acciones. Pero, el acontecimiento es siempre un segmento de un proceso, un corte en el tiempo, un intervalo de proceso entre dos marcas en el tiempo o momentos; y, la acción, una implicación, una participación, un verse complicado en un acontecimiento, en un proceso. Según esto, el quehacer de la educación, ante todo, tiene que ver con comprender acontecimientos. Lo primero que demanda una situación educativa, de cualquier tipo, es aptitud para la comprensión. Si las situaciones educativas, las relacionalmente realizativas, se descortezan y pelan, o se lavan y escamondan, encontramos que el conjunto de sus incidencias, sin ánimo de agotar la descripción, puede agruparse en varias categorías o familias: (i) presencias y ausencias de entes (desde cosas hasta personas), (ii) prácticas y quehaceres (desde cosas que hacer a maneras de proceder), (iii) imaginarios y representaciones (desde imágenes a ilusiones), (iv) discursos y narraciones (desde conversaciones a comentarios textuales) y (v) el chorro de los acontecimientos.

La pedagogía, a diferencia de la historia, que también se ocupa de acontecimientos, por añadidura, está imbuida de propósito, su indagación únicamente se justifica en la medida en la que se implica en lo que queda por acontecer, está empeñada en algo que se quiere que acontezca. En el trabajo pedagógico se asume que, dado un contexto de pasado y el estado de cosas de un "ahora y aquí", siempre pueden darse circunstancias que se constituyan en nexo hacia un plus de calidad de vida posible. Algunos han confundido este nexo y posibilidad con un permanente andar ilusionado, situado en posibles y en valores generales, que permiten una profesión de comodidad infinita, un discurso que siempre acierta, porque trata únicamente con lo que debiera ser o con lo que debiera hacerse.

El punto de mira en lo posible también puede constituir un ejercicio de esperanza y confianza infinita, si considera toda situación como una encrucijada de procesos y trata de encontrar un proyecto, con relación de causalidad apreciable o por lo menos inteligible, entre las circunstancias comprendidas y un margen de posibilidad justificable que oriente la acción. La deliberación pedagógica trata de encontrar la medida en la que la interferencia de la intención, plasmada en planes de acción, puede romper la inercia de un decurso perjudicial, injustificable o insostenible de acontecimientos. De ahí que la pedagogía tenga que construir la parte que le corresponde de la teoría general de la comprensión. Dada la tensión hacia meta (intencionalidad), que alimenta la deliberación pedagógica, no encuentro dificultad alguna en admitir, también, que se emparenta con la amplísima y variada familia de los conocimientos operatorios (técnicos). Es consciente de que su 
intencionalidad es una intencionalidad permanentemente quebrada por las determinaciones y condicionantes de los contextos, está apercibida de que en su ámbito de acción son estrechos los márgenes de eficiencia asegurada. La deliberación pedagógica tiene lugar en sistemas intencionales donde las señales, por las que muestran sus estados las mentes o las comunidades en formación, llegan cargadas de ambigüedad y de incertidumbre. A esa ambigüedad e incertidumbre se encuentra encadenado todo ejercicio de comprensión. Por eso, desde ese momento, adquiere la pedagogía la responsabilidad de construir la parte que le corresponde de la filosofía de la tecnología. Se definen las tecnologías como "sistemas de acciones intencionalmente orientados a la transformación de objetos concretos" (Quintanilla, 1989). Extender esa frontera, para que queden dentro los sistemas de acciones intencionadas, orientados a la modificación de estados de cosas en el comportamiento de agentes intencionales, es objetivo insoslayable de la filosofía de la educación, de la epistemología de la pedagogía. Porque en esos procesos intervienen igualmente aptitudes de inteligencia operatoria. No puede esperar a que se la den hecha. La pedagogía debe investigar las ambigüedades y obscuridades de los nexos entre antecedentes y consecuentes en los procesos de formación, mientras quien delibere, de una u otra manera queda complicado en las prácticas de formación.

\section{¿Cuál es la medida en la que esto es posible en el campo sentimental?}

Una advertencia y precaución es imprescindible antes de seguir. El conocimiento pedagógico, el que se plasma en textos o en discursos, no muestra los acontecimientos vividos, las experiencias privadas, sino su reconstrucción: su reconstitución, empleando conceptos. En la deliberación y en las narrativas no tratamos con acontecimientos, sino con representaciones e interpretaciones de acontecimientos. Las representaciones son los materiales con los que construimos la nueva unidad referenciada, la representación es el material que entra en la fragua de la deliberación. Algunos autores indican que la tarea fundamental de la pedagogía es la reflexión en la acción, durante y mientras el acontecimiento pedagógico acontece, como si los educadores y pedagogos fuesen los únicos para quienes no es legítimo pensar en ausencia del acontecimiento; como si fuesen los únicos agentes intencionales a los que no les fuese reconocido sino actuar o callar. Como si la reflexión pedagógica quedara en mera ficción estratégica en la mente, como si no hubiese otro acceso a los procesos de la educación más que a través del acontecimiento. Si fuese así, para el actor, la unidad de acontecimiento, en su particularidad personal, espacial y temporal, sería siempre incomunicable. Para todo agente intencional, toda deliberación se produce dentro de una fase de suspensión del acontecimiento; es el momento en el que se construye plan, se evalúan consecuencias advertidas, se modifica la estructura del sistema, se va paso a paso, suspendiendo, reflexionando. La acción humana intencional es un "bucle" de acción-suspensión (deliberación)-acción; algo propio, por otra parte, de todos los 
sistemas complejos adaptativos: sistemas en estado de retroalimentación permanente. El protagonista reflexivo o el espectador crítico de escenas, delibera siempre sobre el acontecimiento pasado, mientras y en la medida en la que los acontecimientos van transcurriendo.

La reflexión en ausencia y la crítica a distancia de los acontecimientos es la condición epistémica para toda deliberación. La pretensión de que la pedagogía se reduzca a reflexión en la acción, y al mismo tiempo se constituya en campo de conocimiento, en acumulación de experiencia compartida, es una pretensión imposible. En el acontecimiento, todo es transición, aunque sea propio de la condición humana actuar con conocimiento. Cualquier acontecimiento pedagógico, en el instante en que sufre la metamorfosis de la reflexión y se transforma en objeto de conocimiento es, por lo mismo, una reconstrucción que incorpora conceptos y se imbuye de un mínimo de teoría, queda recreado el acontecimiento en un contexto de conocimiento.

Cuando en la deliberación se hacen intervenir las intenciones, cuando es imposible hacer abstracción de lo que los actores han pensado o querido; en este caso, la forma de deliberación sobre el acontecimiento entra dentro de lo que Max Weber (1984), Dilthey (1940, 1980), G. H. von Wright denominaron la comprensión, señalando que la metodología de la comprensión es bermenéutica, y que la lógica de la comprensión adquiere la apariencia del silogismo práctico. Raymond Aron $^{8}$ indica que aquellas formas de conocimiento, como las históricas, y las que observamos en muchas narrativas pedagógicas, emplean varios enunciados generales, detectan y operan con multiples antecedentes y plantean, también, vínculos causales probables con el acontecimiento. La explicación y la decisión en la práctica se establecen dentro de este régimen de incertidumbre y probabilidad; es una manera, muy humana, de tratar con la causalidad y con la complejidad de manera simultánea. Por lo tanto, en la comprensión del acontecimiento cultural (educacional) particular, situado en el tiempo y el espacio, intervienen, al menos, dos tipos de enunciados generales (teóricos): los que proceden del conocimiento de los observadores respecto al tema, enunciados macroeducacionales y los que proceden de los caracteres personales en la acción de los protagonistas, enunciados disposicionales (caracteres personales en la acción). Algunos entienden que la reflexión en la acción únicamente puede operar con los segundos; pero, no es discutible que, en la práctica, siempre aparecen los primeros aportando inteligibilidad complementaria: basta interrogar en profundidad a los protagonistas.

La comprensión y la hermenéutica han sido los instrumentos de estudio aplicados con mayor antigüedad y de manera más insistente en la actividad con conocimiento de los seres humanos. Especialmente la bermenéutica de la comunicación, que

8. ARON, R. (1996) Lecciones sobre la bistoria. Cursos del Collège de France. México, Fondo de Cultura Económica. Especialmente me refiero al capítulo VIII "La explicación histórica", pp. 135 y ss.; capítulo IX "Explicación histórica y comprensión”, pp. 149 y ss.; capítulo X “Comprensión y narración", pp. 164 y ss. 
emplea como estrategia la introspección, como instrumento expresivo la enorme versatilidad del lenguaje y como táctica de corroboración el consentimiento. Éste ha sido el procedimiento tradicional en la cultura de la sensibilidad, en el rastreo de emociones y sentimientos y en la práctica de su comprensión para inferir consecuencias comportamentales.

\section{La comprensión, la bermenéutica y los mecanismos de los sentimientos}

Pero, la semanticidad del sentimiento y la emoción requiere, además del acontecimiento, de una bermenéutica de la situación y de la escena, no sólo una hermenéutica del vocabulario emocional, también una bermenéutica del movimiento y la seña, una bermenéutica de la forma y sus cambios, una hermenéutica del suceso emotivo en toda su polimórfica identidad; a este nivel de hermenéusis hay que añadir una la bermenéutica de segundo orden para la comprensión de los estados interiores y los metasentimientos (experiencias emocionales acerca de deliberaciones sobre sucesos emocionales), como el desasosiego por haber discutido airado. Lo decimos, no por exagerar, sino por mostrar que en la vía de la sensibilidad el hombre posee complejidad semejante a la complejidad infinita que acepta para la vía cognitiva. Como consecuencia, se producen, también, problemas de interculturalidad y de confrontación en la comprensión de la emocionalidad entre diferentes culturas; aunque, como ya indiqué, hay fondo común entre comunidades culturales diferentes, porque la emocionalidad no es particularidad cultural sino condición humana. Los diferentes artículos de este número de Teoría de la educación constituyen ejemplos y ejercicios de esta hermenéutica de los sentimientos.

Es habitual en las conversaciones identificar la comprensión de un sistema con desentrañar su funcionamiento, con descubrir sus mecanismos. Basta abrir textos de diversas materias para advertir que las palabras "funcionamiento" $\mathrm{y}$ "mecanismo" se emplean en dominios tan diferentes a la mecánica como son el biomolecular, el ecológico, el económico, el del sistema de los sistemas de formación. Nadie piensa en los engranajes de un reloj de cuerda, ni siquiera en uno digital, cuando indica que la distribución de responsabilidad es un mecanismo para aumentar el compromiso institucional o que la dramatización ha sido un mecanismo cultural para la educación de la sensibilidad de colectivos humanos. El término mecanismo está aludiendo en estos casos, como indica M. Bunge, al modus operandi del dinamismo en estos sistemas. Mucho contenido de lo que se ha denominado pedagogía crítica ha consistido precisamente en el desvelamiento de mecanismos de funcionamiento, modos de operar, de las instituciones de formación - sean familias, clases sociales, escuelas o Estados-. P. Bourdieu y J. C. Passeron (1977) trataron de demostrar que el mecanismo encubierto de toda acción pedagógica (proceso, sistema) —en la familia, la ciudad o la escuela-, consistía en una dinámica que empleaba la inculcación, con violencia simbólica, para imponer una arbitrariedad cultural. Muchos de los denominados procedimientos pedagógicos 
formulados como criterios de actuación constituyen enunciados que sospechan un mecanismo encubierto, el cual intentan aprovechar intencionalmente para que acontezcan cambios en los estados de cosas.

El estudio de la estructura del sistema emocional proporciona evidencias de la existencia de planos en la sensibilidad y, por lo tanto, evidencia de varias categorías de mecanismos. J. Morgan Allman (2003), por ejemplo, señala estos niveles: (i) reserva el término emocional para el subcortical y (ii) adjudica el término sentimental para el nivel cortical asociado a la consciencia. Por otro lado, las neurociencias informan de la existencia de (i) sentimientos de fondo, asociados, entre otros estados de subsistemas, al tono muscular (Llinás, 2003), p. e., la denominada sensación de bienestar; (ii) concuerdan, igualmente, los neurólogos en indicar la existencia de un grupo limitado de emociones básicas cuya denominación varía, de manera comprensible, de unos autores a otros (Damasio, 2001), porque no para todos las mismas palabras aluden al referente que sospechan. Tanto una categoría como otra debieran estudiarse no partiendo de la semanticidad lingüistica, por su ambigüedad, sino por las funciones adaptativas que cumplen las tramas emocionales básicas; es más conveniente estudiarlas en la práctica y en los acontecimientos que en los diccionarios. A partir de ahí, tomando los elementos constituyentes de las tramas emocionales -escenarios y escenas, objetos referenciales, síntomas corporales, estados internos, memoria emocional, actitudes relacionales con los objetos (sorpresa, pérdida, reencuentro...), calidades e intensidades de apego, la positividad y negatividad respecto a la dinámica experiencial del sujeto... - se puede concebir el crecimiento y el incremento arborescente de la emocionalidad al que aludía el Dalai Lama refiriéndose a las narrativas budistas, los "miles" de tipos emocionales (Dalai Lama, 2004, 37).

No debe sorprender el hecho de proponer un limitado número de sentimientos de fondo y de emociones básicas, manteniendo el crédito debido a la experiencia que muestra una infinidad de emociones secundarias. También son 28 los fonemas del alfabeto castellano, y cinco las clases de papilas gustativas en la lengua - para notar los cinco sabores básicos-, como son limitados los grados de libertad de las articulaciones; cada uno de esos sistemas genera complejidad ilimitada en la expresividad oral, una sinfonía de sabores, un sistema completo de comunicación por la seña y una enorme variedad de matices en el discernimiento valorado de las situaciones interiores y exteriores a través del sentimiento. A. Damasio no cree, por motivos fisiológicos, que ni el dolor ni el placer sean emociones, aunque son inductores poderosos de variedades emocionales sin límite; pero esta cuestión discutible no perjudica a la totalidad del planteamiento.

En la literatura emocional el término sentimiento, categoría emocional diferente a las anteriormente aludidas, se trata a veces, en tan exquisitos términos que, al final, como en Descartes, terminan teniendo su sede en el "alma". Confieso mi predilección por el planteamiento general de A. Damasio (2005, 84 y ss.) en este punto, en su reciente obra En busca de Spinoza que, por otra parte, recapitula el contenido básico de sus obras anteriores. Veamos en un breve resumen los elementos 
de su modelo. No deja que el marco de referencia deje de ser el cuerpo: se pregunta por "la materia del pensamiento". El sentimiento es suceso, estado, en la unidad psicosomática. A los sentimientos también les alcanza la marcación somática. Los componentes principales del estado sentimental serían los siguientes: (i) la materia del sentimiento está formada por la representación en el cerebro de estados corporales; (ii) el sentimiento arranca de la percepción consciente de un estado particular del cuerpo, "surgen de cualquier conjunto de reacciones homeostáticas, no únicamente de las emociones propiamente dichas"; (iii) acoplamiento de pensamientos concordes con el estado del sistema emocional, "un estilo de procesamiento mental".

En este modelo los sentimientos son, ante todo, estados perceptivos ("percepciones"), cuyo recorrido transcurre en el interior del sujeto, culminando como atractor de pensamientos afines; las emociones asumirían el carácter de estados activos (acciones) en respuesta a los inductores situacionales del entorno ("estímulos emocionalmente competentes").

Como se puede comprobar el horizonte de la emocionalidad es tan complejo como el panorama de la racionalidad. La razón tiene que bragarse para la comprensión y la explicación, para la investigación de la complejidad de la ontología emocional. Creo que una pedagogía de la sensibilidad debiera tomar en cuenta esa conexión activa con el exterior que mantienen las emociones, a la hora de fomentarlas y cultivarlas; como también debiera estar alerta a esa trayectoria interior del sentimiento a la hora de enseñar a "leer" en la sentimentalidad, corregir su ortografía o tratar de promover "nuevas experiencias lectoras".

\section{BiBLIOGRAFíA}

Aguado, L. (2005) Emoción, afecto y motivación. Madrid, Alianza.

Aguirre Sala, J. F. (2005) Hermenéutica ética de la pasión. Salamanca, Sígueme.

BOURDIEU, P. y PASSERON, J. C. (1977) La reproducción. Elementos para una teoria del sistema de enseñanza. Barcelona, Laia.

Breton, D. Le (2004) Les passions ordinaires. Antbropologie des émotions. París, Payot.

BRUNER, J. (1988) Realidad mental y mundos posibles. Los actos de la imaginación que dan sentido a la experiencia. Barcelona, Gedisa.

Bunge, M. (2000) La relación entre la sociología y la filosofía. Madrid, EDAF-ensayo.

Buss, D. M. (1994) La evolución del deseo. Estrategias del emparejamiento bumano. Madrid, Alianza.

Camps, V. (2001) Una vida de calidad. Reflexiones sobre la bioética. Barcelona, Ares y Mares.

Cannon, W. B. (1946) La sagesse du corps. París, Nouvelle Revue Critique.

Casanova, G. (1993) Mémoires: histoire de ma vie. París, Arlea.

Castilla del Pino, C. (2000) Teoría de los sentimientos. Barcelona, Tusquets.

CaVallé, M. (2004) La filosofia maestra de vida. Respuestas a las inquietudes de la mujer de boy. Madrid, Aguilar.

Dalai LAMA (2004) Con el corazón abierto. El mensaje inspirador de un hombre sabio. Barcelona, Debolsillo. 
DAMASIO, A. (1996) El error de Descartes. La emoción, la razón y el cerebro bumano. Barcelona, Grijalbo-Mondadori.

- (2001) La sensación de lo que ocurre. Cuerpo y emoción en la construcción de la conciencia. Madrid, Debate.

- (2005) En busca de Spinoza. Neurobiología de la emoción y los sentimientos. Barcelona, Crítica.

DAY, Ch. (2006) Pasión por enseñar. La identidad personal y profesional del docente y sus valores. Madrid, Narcea.

DennetT, D. C. (2000) Tipos de mentes. Hacia una comprensión de la conciencia. Barcelona, Debate.

DiltheY, W. (1940) Fundamentos de un sistema de pedagogía. Buenos Aires, Losada.

- (1980) Introducción a las ciencias del espiritu: ensayo de una fundamentación del estudio de la sociedad y de la historia. Madrid, Alianza.

Duch, L. y MÈııCH, J. C. (2005) Escenarios de la corporeidad: antropología de la vida cotidiana. Madrid, Trotta.

Ekman, P. (2004) ¿Qué dice ese gesto? Barcelona, RBA.

Evans, D. (2002) Emoción. La ciencia del sentimiento. Madrid, Taurus.

FLAUBERT, G. (1990) La educación sentimental. Madrid, Cátedra.

FRIED (1995) The passionate teacher: a practical guide. Boston, Beacon Press.

García Carrasco, J. (2004) La comprensión de la vivienda como un dominio vital de los humanos, Revista Española de Pedagogía, n. ${ }^{\circ} 288$ (LXII), 229-256.

GARDNER, H. (1994) Estructura de la mente. La teoria de las inteligencias múltiples. México, Fondo de Cultura Económica.

Gil Roales-Nieto, J. y López Ríos, F. (2004) Estrés y salud, en Psicología de la salud. Aproximación bistórica, conceptual y aplicaciones. Barcelona, Pirámide, 351 y ss.

Godwin, G. (2004) El corazón. Itinerario por los Mitos y Significados. Madrid, Espasa.

Goleman, D. (1996) Inteligencia emocional. Barcelona, Kairós.

- (1997) El punto ciego. Psicología del autoengaño. Barcelona, Plaza y Janés.

- (1999) La práctica de la inteligencia emocional. Barcelona, Kairós.

- (2003) Emociones destructivas. Barcelona, Kairós.

GREENSPAN, S. I. y THORNDIKE-GREENSPAN, N. (1997b) Las primeras emociones. Barcelona, Paidós. Grewal, D. y Salovey, P. (2006) Inteligencia emocional, Rv. Mente y Cerebro, n. ${ }^{\circ} 16,10$ y ss.

HuMPHREY, N. (1995) Una bistoria de la mente. La evolución y el nacimiento de la conciencia. Barcelona, Gedisa.

JONAS, H. (1995) El principio de responsabilidad. Ensayo de una ética para la civilización tecnológica. Barcelona, Herder.

- (1997) Técnica, medicina y ética. La práctica del principio de responsabilidad. Barcelona, Paidós.

- (2000) El principio vida. Hacia una biología filosófica. Valladolid, Trotta.

JuAN DE LA CRUz (1993) Cántico espiritual. Estudio preliminar de Víctor García de la Concha. Madrid, Hipánica de Bibliofilia.

Kramer, S. (1978) La bistoria empieza en Sumer. Barcelona, Aymá.

LAZARus, R. S. y LAZARus, B. N. (2000) Pasión y razón. La comprensión de nuestras emociones. Barcelona, Paidós.

LEWIS, Th.; AMINI, F. y LANNON, R. (2001) Una teoria general del amor. Barcelona, RBA.

LuNAS, R. R. (2003) El cerebro y el mito del yo. El papel de las neuronas en el pensamiento y el comportamiento bumanos. Barcelona, Belacqua. 
LuTz, T. (2001) El llanto. Historia cultural de las lágrimas. Madrid, Taurus.

MARÍAs, J. (1992) La educación sentimental. Madrid, Alianza.

MARINOFF, L. (2000) Más Platón y menos Prozac. Barcelona, Ediciones B.

Matignon, K. L. (2005) Émotions animales. París, É. du Chêne-Hachette.

Miller, W. I. (1998) Anatomía del asco. Madrid, Taurus.

Morgado, I. (ed.) (2002) Emoción y conocimiento. Barcelona, Metatemas.

Morgan Allman, J. (2003) El cerebro en evolución. Barcelona, Ariel.

Nussbaum, M. C. (2003) La terapia del deseo. Teoría y práctica de la ética belenística. Barcelona, Paidós.

- (2005) El conocimiento del amor. Ensayos sobre filosofía y literatura. Madrid, A. Machado libros.

ORNSTEIN, R. (1994) La evolución de la conciencia. Los limites del pensamiento racional. Barcelona, Emecé.

PICARD, R. (1998) Los ordenadores emocionales. Barcelona, Ariel.

- (2002) Los ordenadores emocionales, en Morgado, I. (ed.). Emoción y conocimiento. Barcelona, Metatemas, 165 y ss.

PINKER, S. (2000) Cómo funciona la mente. Barcelona, Destino.

Polaino, A. (2003) Familia y autoestima. Barcelona, Ariel.

Quintanilla, M. A. (1989) Tecnología. Un enfoque filosófico. Madrid, Fundesco.

RougEMONT, D. (1993 v.o. 1978) El amor y Occidente. Barcelona, Kairós.

RYLE, G. (1967 v.o. 1949) El concepto de lo mental. Buenos Aires, Paidós.

SeOANe Pinilla, J. (2004) Del sentido moral a la moral sentimental. El origen sentimental de la identidad y la ciudadania democrática. Madrid, Siglo XXI.

SERVAN-SCHREIBER, D. (2004) Curación emocional. Acabar con el estrés, la ansiedad y la depresión sin fármacos ni psicoanálisis. Barcelona, Kairós.

Slater, P. J. B. (2000) El comportamiento animal. Madrid, Cambridge Univ. Press.

Smith, A. (1997) Teoría de los sentimientos morales. Madrid, Alianza.

Speranza, M. y Atger, F. (2003) Approche développemental de l'alexithymie, en Corcos, M. (ed.). Psycopathologie de l'alexithymie. París, Dunod, 65-80.

SPINOZA, B. (1988) Ética. Madrid, Alianza Editorial.

TAYLOR, Sh. E. (2002) Lazos vitales. De cómo el cuidado y el afecto son esenciales para nuestras vidas. Madrid, Taurus.

TRAPPI, R.; PETTA, P. y PAYR, S. (2003) Emotions in Humans and Artifacts. Massachussets, MIT Press.

VARELA, F. (1992) De cuerpo presente. Las ciencias cognitivas y la experiencia bumana. Barcelona, Gedisa.

Vigotsky, L. S. (1972) Psicologia del arte. Barcelona, Barral.

VINCENT, J. D. (1987) Biología de las pasiones. Barcelona, Anagrama.

WAAL, F. (1993) La Política de los chimpancés. El poder y el sexo entre los simios. Madrid, Alianza.

- (2002) El simio y el aprendiz de Sushi. Reflexiones de un primatólogo sobre la cultura. Barcelona, Paidós.

WeBER, M. (1984) La acción social: ensayos metodológicos. Barcelona, Península.

WILSON, E. O. (1980) Sobre la naturaleza bumana. Madrid, Fondo de Cultura Económica.

WRIGTH, G. H. (1980) Explicación y comprensión. Madrid, Alianza.

Zamora, J. M. (2003) Las pasiones del Pórtico. Concepciones del páthos en el estoicismo antiguo y medio, Cuaderno Gris, Epoca III, n. ${ }^{\circ}, 23$ y ss.

ZELDIN, Th. (1996) Historia intima de la bumanidad. Madrid, Alianza. 